\section{PWE-111 IMPACT OF THE JAG BASIC SKILLS IN COLONOSCOPY COURSE ON TRAINEE PERFORMANCE}

1,2 Keith Siau*, ${ }^{2}$ James Hodson, ${ }^{1,3}$ John T Anderson, ${ }^{3}$ Roland Valori, ${ }^{1,4}$ Paul Hagan, 1,5Geoff Smith, ${ }^{1,3}{ }^{3}$ Paul Dunckley. ${ }^{1} J o i n t$ Advisory Group on Gastrointestinal Endoscopy (JAG), London; ${ }^{2}$ University of Birmingham, Birmingham; ${ }^{3}$ Gloucester Hospitals NHSFT; ${ }^{4}$ King's Mill Hospital, Mansfield; Imperial College NHSFT, London

\subsection{6/gutjnl-2019-BSGAbstracts.482}

Introduction The JAG Basic Skills in Colonoscopy Course is a mandated JAG course designed to help standardise colonoscopy training within the UK. The course is a component of the certification criteria, but the impact of the course on subsequent trainee performance is unknown. We aimed to evaluate colonoscopy performance, as measured using colonoscopy completion metrics, in relation to course attendance.

Methods Trainees awarded colonoscopy certification between 201-016 were stratified into 3 groups according to the number of procedures performed prior to the course, $<70,7-40$ and $>140$ procedures. The study outcomes comprised the unassisted caecal intubation rate (CIR) and the Performance Indicator of Colonic Intubation (PICI), ${ }^{1}$ a composite measure encompassing caecal intubation, sedation and discomfort. Within each group, outcomes rates were calculated for each of the 50 procedures before and after the course. Interrupted time series models were then used to detect step-change improvements occurring after the course.

Results A total of 369 trainees were included in the analysis, who performed $<70 \quad(\mathrm{~N}=118), 7-40 \quad(\mathrm{~N}=121)$ or $>140$ $(\mathrm{N}=130)$ procedures prior to the course. Over the 50 procedures prior to the course, all three groups saw significant linear improvements in CIR, with an average increase of 4.2, 3.6 and 1.7 percentage points for every 10 procedures in the $<70,7-40$ and $>140$ groups, respectively (all $\mathrm{p}<0.001$ ). In those with $<70$ procedures at the time of the course, a significant step-change improvement in CIR was detected, from $46 \%$ in the last procedure before the course, to $51 \%$ in the first procedure afterwards $(p=0.005)$. No significant improvement in CIR was detected in the other two groups, with
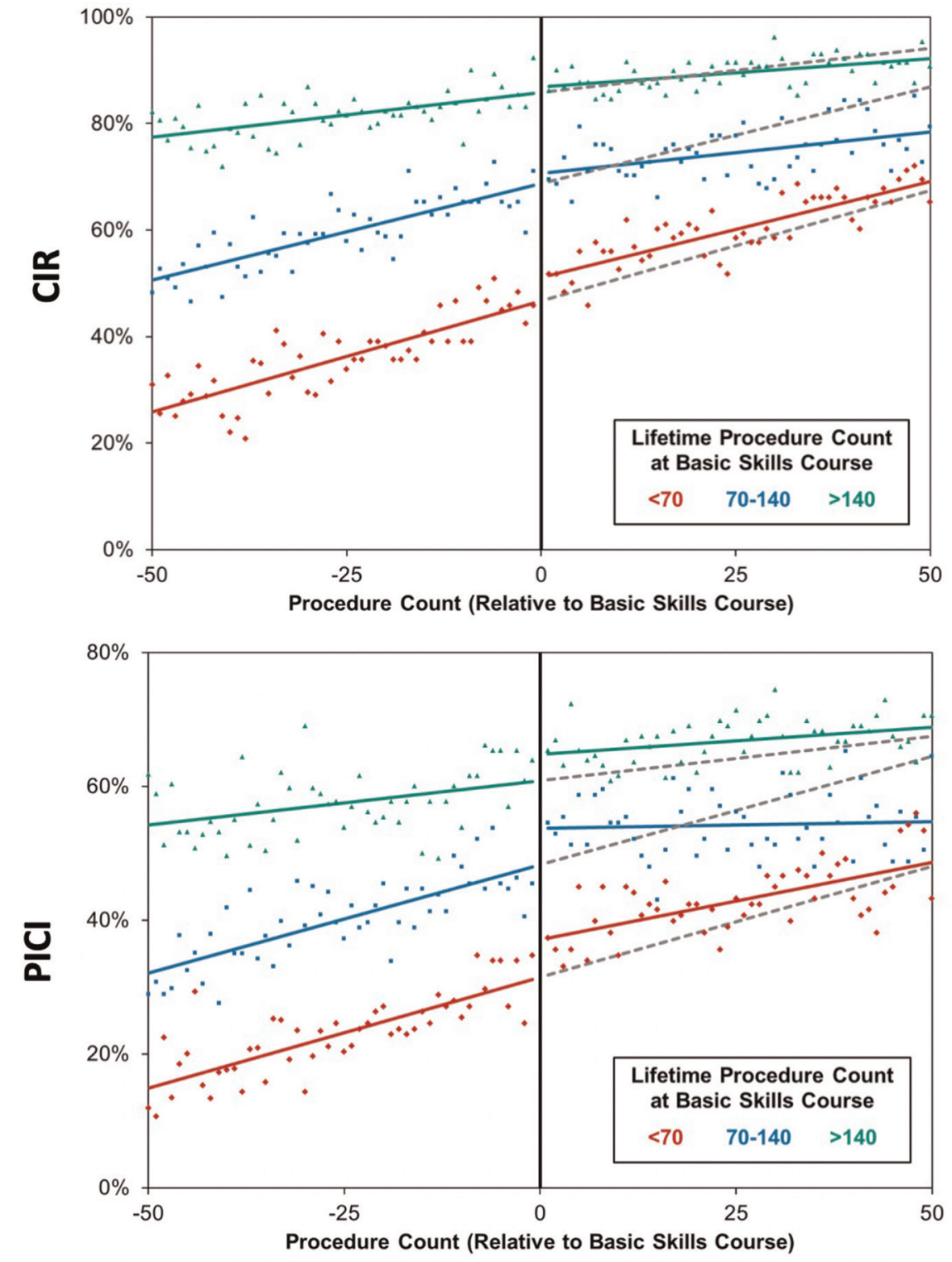
changes of $68 \%$ to $71 \% \quad(\mathrm{p}=0.239)$ and $86 \%$ to $87 \%$ $(p=0.354)$ for the $7-40$ and $>140$ procedure groups, respectively. For PICI, all three groups saw a significant step change improvement, with average increases of $5.6(\mathrm{p}<0.001), 5.4$ $(p=0.003)$ and $3.9(p=0.014)$ percentage points for the $<70$, $7-40$ and $>140$ groups, respectively. Based on pre-course trends, the immediate improvement in PICI following course attendance was equivalent to that stemming from performing an additional 1-0 procedures.

Conclusions Attendance of the JAG Basic Skills Colonoscopy Course appears to improve PICI in all trainees. CIR data suggests that the optimal timing of course attendance appears to be at earlier stages of colonoscopy training ( $<70$ procedures).

\section{REFERENCES}

2. Valori RM, et al. Endoscopy 2018; PMID:28753700

\section{PWE-112 ERCP DOPS ASSESSMENTS: EVIDENCE OF VALIDITY AND COMPETENCY DEVELOPMENT DURING TRAINING}

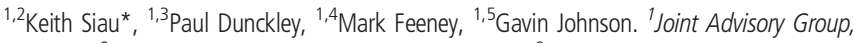
London; '2University of Birmingham, Birmingham; ${ }^{3}$ Gloucestershire Royal Hospital, Gloucester; ${ }^{4}$ Torbay and South Devon NHSFT, Torquay; ${ }^{5}$ University College London Hospitals NHSFT, London

\subsection{6/gutjnl-2019-BSGAbstracts.483}

Introduction Formative direct observation of procedural skills (DOPS) in ERCP consist of 27 assessable items located within 7 domains and an overall competence rating. Despite their implementation in 2016, validity evidence remain lacking. We aimed to evaluate DOPS scores to appraise validity and competency development during ERCP training.

Methods This prospective UK-wide study analysed ERCP DOPS submitted to the JETS e-portfolio between July 2016October 2018. Reliability was measured using Cronbach's alpha. DOPS scores were benchmarked using the contrasting groups method to establish consequential validity. The percentage of competent scores were averaged for each item, domain and overall rating and stratified by lifetime procedure count to plot learning curves and provide discriminative validity. Multivariable generalising estimating equations were performed to identify trainee-level predictors of overall procedural competence.

Results In total, 818 DOPS submitted from 80 UK centres were analysed. DOPS were completed for 109 trainees (ST-: 26\%, ST6: 22\%, ST-: 21\%, research fellow: 7\%, consultant/ associate specialist: 24\%). 5 items were unassessed in $>50 \%$ of DOPS and were excluded from Cronbach's alpha analyses; assessment of the remaining 22 items yielded a statistic of 0.95 , indicating high reliability. Attaining competency in $87 \%$ of assessed items per DOPS provided the optimal benchmark score (false positive: 10\%; false negative: 2\%). Competency acquisition occurred in the domain sequence of: 'pre-procedural', 'intubation and positioning', 'post-procedural', 'endoscopic non-technical skills', 'execution of selected therapy', and 'cannulation and imaging' (Figure). Trainees surpassed the $87 \%$ competency threshold after $20-49$ procedures (mean: 89\%). After 300 procedures, the competency threshold was reached for 'selective cannulation' (89\%), but not reached for the items of: stenting (plastic: 73\%; metal: 70\%), sphincterotomy $(80 \%)$ and sphincteroplasty $(56 \%)$. On multivariable analysis, lifetime procedure count $(\mathrm{P}<0.001)$, easier case difficulty $(\mathrm{P}<0.001)$ and lifetime DOPS count $>10 \quad(\mathrm{P}=0.002)$ predicted overall procedural competence, but not trainee specialty $(\mathrm{P}=0.525)$, grade $(\mathrm{P}=0.076)$ or prior gastroscopy certification $(\mathrm{P}=0.886)$.

Conclusion This study provides novel validity, reliability and learning curve data in support of ERCP DOPS. Whilst competency in the majority of DOPS items may be attained after 20-49 procedures, this may still be insufficient for selective cannulation and higher-level therapeutic competencies.

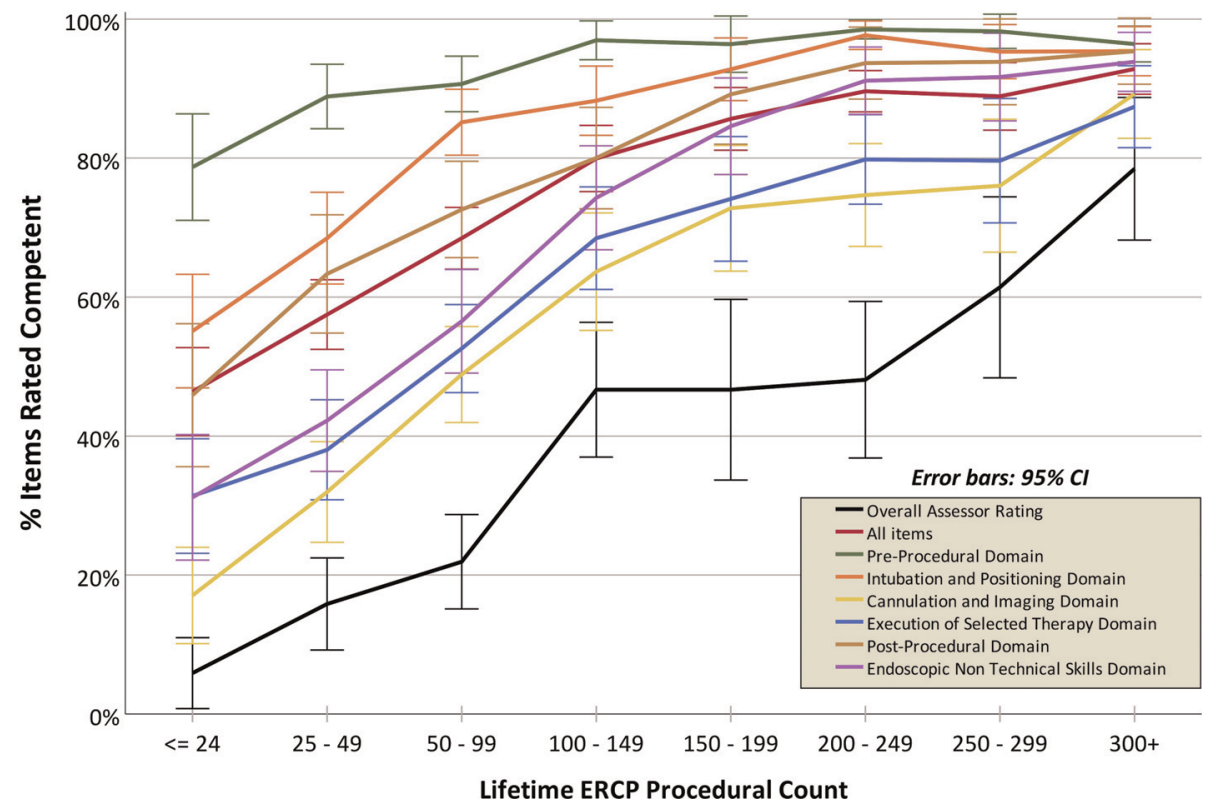

Abstract PWE-112 Figure 1 